PROCEEDINGS OF THE

AMERICAN MATHEMATICAL SOCIETY

Volume 132, Number 10, Pages 2959-2967

S 0002-9939(04)07579-3

Article electronically published on June 2, 2004

\title{
WEAK SPECTRAL SYNTHESIS FOR THE PROJECTIVE TENSOR PRODUCT OF COMMUTATIVE BANACH ALGEBRAS
}

\author{
EBERHARD KANIUTH
}

(Communicated by David R. Larson)

\begin{abstract}
Let $A$ and $B$ be regular commutative Banach algebras and $A \widehat{\otimes} B$ their projective tensor product, and suppose that $A \widehat{\otimes} B$ is semisimple. We investigate the relation between weak spectral synthesis properties of $A \widehat{\otimes} B$ and those of $A$ and $B$.
\end{abstract}

\section{INTRODUCTION}

Let $A$ be a semisimple regular commutative Banach algebra with structure space $\Delta(A)$ and Gelfand transform $a \rightarrow \widehat{a}$. For any subset $M$ of $A$, the hull $h(M)$ of $M$ is defined by $h(M)=\{\varphi \in \Delta(A): \varphi(M)=\{0\}\}$. Associated to each closed subset $E$ of $\Delta(A)$ is a largest and a smallest ideal, $k(E)$ and $j(E)$, of $A$ with hull equal to $E$, namely

$$
k(E)=\{a \in A: \widehat{a}(\varphi)=0 \text { for all } \varphi \in E\}
$$

and

$$
j(E)=\{a \in A: \widehat{a} \text { has compact support disjoint from } E\} .
$$

The set $E$ is called a spectral set (or set of spectral synthesis) if $k(E)=\overline{j(E)}$ (equivalently, $k(E)$ is the only closed ideal of $A$ with hull equal to $E$ ). We say that spectral synthesis holds for $A$ if every closed subset of $\Delta(A)$ is a spectral set.

A weaker form of spectral synthesis has been introduced in [14]. A closed subset $E$ of $\Delta(A)$ is called a weak spectral set if every element of the quotient algebra $k(E) / \overline{j(E)}$ is nilpotent. When this happens for each $E$, we say that weak synthesis holds for $A$. The importance of these latter notions is due to the fact that the class of weak spectral sets is usually considerably larger than the class of spectral sets and that there are many commutative Banach algebras for which weak synthesis holds, but synthesis fails.

Now, the structure space of the projective tensor product $A \widehat{\otimes} B$ of two commutative Banach algebras $A$ and $B$ is canonically homeomorphic to the product space $\Delta(A) \times \Delta(B)$. More precisely, given $\varphi \in \Delta(A)$ and $\psi \in \Delta(B)$, there is a unique homomorphism $\varphi \widehat{\otimes} \psi: A \widehat{\otimes} B \rightarrow \mathbb{C}$ such that $\varphi \widehat{\otimes} \psi(a \otimes b)=\varphi(a) \psi(b)$ for all $a \in A$ and $b \in B$, and the map $(\varphi, \psi) \rightarrow \varphi \widehat{\otimes} \psi$ is a homeomorphism between the product space $\Delta(A) \times \Delta(B)$ and $\Delta(A \widehat{\otimes} B)[4,11$. Nevertheless, surprisingly little is known about relating spectral synthesis problems in $A \widehat{\otimes} B$ to such problems in $A$ and $B$.

Received by the editors February 3, 2003.

2000 Mathematics Subject Classification. Primary 46J20, 46J05. 
In this paper we show that, under some standard condition on $A$ and $B$, a product subset $E \times F$ of $\Delta(A \widehat{\otimes} B)$ is a weak spectral set for $A \widehat{\otimes} B$ if and only if $E$ and $F$ are weak spectral sets for $A$ and $B$, respectively, and similarly for spectral sets (Theorems 1.2 and 1.6). Moreover, when $\Delta(B)$ is discrete, we characterize the properties of spectral synthesis and weak spectral synthesis for $A \widehat{\otimes} B$ in terms of the corresponding properties for $A$ (Theorem 2.2). An application (Corollary 2.3) concerns generalized $L^{1}$-group algebras.

\section{WEAK SPECTRAL SETS OF PRODUCT TYPE}

For two complex algebras $A$ and $B, A \otimes B$ will denote their algebraic tensor product, and if $A$ and $B$ are Banach algebras, $A \widehat{\otimes} B$ denotes their projective tensor product. Note that when $A$ and $B$ are commutative Banach algebras, then $A \widehat{\otimes} B$ is regular if and only if both $A$ and $B$ are regular, and if $A \widehat{\otimes} B$ is semisimple, then so are $A$ and $B$. The converse is false in general. In fact, this was shown in 7 , Theorem 3 ] using the existence of Banach spaces that do not have the approximation property 3]. However, if at least one of $A$ and $B$ does have the approximation property and $A$ and $B$ are semisimple, then $A \widehat{\otimes} B$ is semisimple.

Let $A$ be a semisimple regular commutative Banach algebra, and let $E \subseteq \Delta(A)$ be a weak spectral set. Then there exists $n \in \mathbb{N}$ such that $a^{n} \in \overline{j(E)}$ for all $a \in k(E)$ (see [2, footnote 7, p. 855] and [14, Theorem 1.2]). Since $k(E) / \overline{j(E)}$ is commutative, it follows that every $n$-fold product of elements in $k(E)$ belongs to $\overline{j(E)}$. Adopting the notation of [14], we let $\xi(E)$ denote the smallest such number $n$. So $E$ is a spectral set precisely when $\xi(E)=1$.

For $\psi \in \Delta(B)$, let $h_{\psi}: A \widehat{\otimes} B \rightarrow A$ denote the homomorphism satisfying $h_{\psi}(a \otimes$ $b)=\psi(b) a$ for all $a \in A$ and $b \in B$. Note that $\left\|h_{\psi}\right\| \leq 1$. The following simple fact will be used several times.

Lemma 1.1. Let $E$ and $F$ be closed subsets of $\Delta(A)$ and $\Delta(B)$, respectively, and let $\psi \in F$. Then

$$
h_{\psi}(j(E \times F)) \subseteq j(E) .
$$

Proof. Let $x \in j(E \times F)$. There exists a compact subset $C$ of $\Delta(A \widehat{\otimes} B)=\Delta(A) \times$ $\Delta(B)$ such that $\widehat{x}=0$ outside of $C$ and $C \cap(E \times F)=\emptyset$. Thus, if $\varphi \in \Delta(A)$ is such that $0 \neq \varphi\left(h_{\psi}(x)\right)=(\varphi \widehat{\otimes} \psi)(x)$, then $\varphi \widehat{\otimes} \psi \in C$ and $\varphi \notin E$ since otherwise $\varphi \widehat{\otimes} \psi \in C \cap(E \times F)$. So $\widehat{h_{\psi}(x)}$ has compact support disjoint from $E$, whence $h_{\psi}(x) \in j(E)$.

Theorem 1.2. Let $A$ and $B$ be regular commutative Banach algebras, and suppose that $A \widehat{\otimes} B$ is semisimple. Let $E$ and $F$ be closed subsets of $\Delta(A)$ and $\Delta(B)$, respectively. If $E \times F$ is a weak spectral set for $A \widehat{\otimes} B$, then $\xi(E) \leq \xi(E \times F)$, so that $E$ is a weak spectral set for $A$. In particular, if $E \times F$ is a spectral set for $A \widehat{\otimes} B$, then $E$ is a spectral set for $A$.

Proof. Let $n=\xi(E \times F)$, and let $a \in k(E)$ and $\epsilon>0$ be given. Choose $\psi \in F$ and $b \in B$ such that $\psi(b)=1$. Since $k(E \times F)^{n} \subseteq \overline{j(E \times F)}$, there exists $x \in j(E \times F)$ such that $\left\|x-(a \otimes b)^{n}\right\| \leq \epsilon$. Then

$$
\begin{aligned}
\left\|a^{n}-h_{\psi}(x)\right\| & =\left\|\psi\left(b^{n}\right) a^{n}-h_{\psi}(x)\right\|=\left\|h_{\psi}\left(a^{n} \otimes b^{n}\right)-h_{\psi}(x)\right\| \\
& \leq\left\|a^{n} \otimes b^{n}-x\right\| \leq \epsilon,
\end{aligned}
$$


and $h_{\psi}(x) \in j(E)$ by the preceding lemma. Since $\epsilon>0$ is arbitrary, it follows that $a^{n} \in \overline{j(E)}$.

To establish the converse to Theorem 1.2 requires some more effort.

Lemma 1.3. Let $A$ and $B$ be commutative Banach algebras, and suppose that $B$ is semisimple. Let $E$ be a closed subset of $\Delta(A)$. Then

(i) $k(E \times \Delta(B))=k(E) \widehat{\otimes} B$;

(ii) $j(E \times \Delta(B)) \subseteq \overline{j(E) \otimes B} \subseteq \overline{j(E \times \Delta(B))}$.

Proof. Let $x=\sum_{i=1}^{\infty} a_{i} \otimes b_{i} \in A \widehat{\otimes} B$, and let $\varphi \in \Delta(A)$ be such that $\varphi \widehat{\otimes} \psi(x)=0$ for all $\psi \in \Delta(B)$. Of course, we can assume that the elements $b_{i}, i \in \mathbb{N}$, are linearly independent. We are going to show that $a_{k} \in \operatorname{ker} \varphi$ for all $k \in \mathbb{N}$. For each $\psi \in \Delta(B)$, we have

$$
\psi\left(\sum_{i=1}^{\infty} \varphi\left(a_{i}\right) b_{i}\right)=\sum_{i=1}^{\infty} \varphi\left(a_{i}\right) \psi\left(b_{i}\right)=\varphi \widehat{\otimes} \psi(x)=0,
$$

whence $\sum_{i=1}^{\infty} \varphi\left(a_{i}\right) b_{i}=0$ since $B$ is semisimple. For every $f \in B^{*}$, we get that

$$
\varphi\left(\sum_{i=1}^{\infty} f\left(b_{i}\right) a_{i}\right)=\sum_{i=1}^{\infty} f\left(b_{i}\right) \varphi\left(a_{i}\right)=f\left(\sum_{i=1}^{\infty} \varphi\left(a_{i}\right) b_{i}\right)=0 .
$$

Fix $k \in \mathbb{N}$ and for $n>k$, let $L_{n}$ denote the linear subspace of $B$ generated by $b_{1}, \ldots, b_{k-1}, b_{k+1}, \ldots b_{n}$. Then $b_{k} \notin L_{n}$, and hence by the Hahn-Banach Theorem there exists $f_{n} \in B^{*}$ such that $f_{n} \mid L_{n}=0,\left\|f_{n}\right\|=1$ and $f_{n}\left(b_{k}\right)=\left\|b_{k}\right\|$. Then, for each $n>k$,

$$
0=\varphi\left(\sum_{i=1}^{\infty} f_{n}\left(b_{i}\right) a_{i}\right)=\left\|b_{k}\right\| \varphi\left(a_{k}\right)+f_{n}\left(\sum_{i=n+1}^{\infty} \varphi\left(a_{i}\right) b_{i}\right) .
$$

Now, since $\sum_{i=1}^{\infty}\left\|a_{i}\right\|\left\|b_{i}\right\|<\infty$ and $\left\|f_{n}\right\|=1$,

$$
\begin{aligned}
\left|f_{n}\left(\sum_{i=n+1}^{\infty} \varphi\left(a_{i}\right) b_{i}\right)\right| & \leq\left\|\sum_{i=n+1}^{\infty} \varphi\left(a_{i}\right) b_{i}\right\| \leq \sum_{i=n+1}^{\infty}\left|\varphi\left(a_{i}\right)\right| \cdot\left\|b_{i}\right\| \\
& \leq \sum_{i=n+1}^{\infty}\left\|a_{i}\right\| \cdot\left\|b_{i}\right\|,
\end{aligned}
$$

which converges to 0 as $n \rightarrow \infty$. It follows that $\varphi\left(a_{k}\right)=0$. Since this holds for all $k$, we conclude that $x \in \operatorname{ker} \varphi \widehat{\otimes} B$. Thus $k(E \times \Delta(B)) \subseteq k(E) \widehat{\otimes} B$. The converse inclusion being obvious, we obtain (i).

Now, assume that $x \in j(E \times \Delta(B))$. There exists a compact subset $C$ of $\Delta(A)$ such that $C \cap E=\emptyset$ and $\varphi \widehat{\otimes} \psi(x)=0$ for all $\varphi \in \Delta(A) \backslash C$ and $\psi \in \Delta(B)$. By what we have seen above, $a_{k} \in k(\Delta(A) \backslash C) \subseteq j(E)$ for all $k \in \mathbb{N}$. Consequently, $x \in \overline{j(E) \otimes B}$.

Finally, if $x=\sum_{i=1}^{m} a_{i} \otimes b_{i}$ is such that $a_{i} \in j(E)$ for all $i$, then $a_{i} \otimes b_{i} \in$ $j(E \times \Delta(B))$ for each $i$. This completes the proof of (ii).

Corollary 1.4. Let $A$ and $B$ be regular commutative Banach algebras such that $A \widehat{\otimes} B$ is semisimple. Let $E$ be a closed subset of $\Delta(A)$, and suppose that $n=\xi(E)<$ $\infty$. Then

$$
k(E \times \Delta(B))^{n} \subseteq \overline{j(E \times \Delta(B))} .
$$


In particular, if $E$ is a set of synthesis for $A$, then $E \times \Delta(B)$ is a set of synthesis for $A \widehat{\otimes} B$.

Proof. Since $A \widehat{\otimes} B$ is semisimple, $j(E \times \Delta(B))$ is the smallest ideal of $A \widehat{\otimes} B$ with hull equal to $E \times \Delta(B)$, and similarly for $j(E)$ since $A$ is semisimple. Therefore, the hypotheses and Lemma 1.3 yield that

$$
\begin{aligned}
k(E \times \Delta(B))^{n} & =(k(E) \widehat{\otimes} B)^{n} \subseteq \overline{k(E)^{n}} \widehat{\otimes} B \\
& \subseteq \overline{j(E)} \widehat{\otimes} B \subseteq \overline{j(E \times \Delta(B))} .
\end{aligned}
$$

The last statement follows with $n=1$.

Before proceeding we recall that a commutative Banach algebra $A$ is said to be Tauberian if the ideal $j(\emptyset)=\{a \in A$ : $\widehat{a}$ has compact support $\}$ is dense in $A$. Thus $A$ is Tauberian exactly when $\emptyset$ is a spectral set. Moreover, $A$ satisfies Ditkin's condition at infinity if $a \in \overline{a j(\emptyset)}$ for all $a \in A$. For these and other notions in spectral synthesis and ideal theory we refer to [6].

Lemma 1.5. Suppose that $A$ and $B$ are Tauberian, and let $E$ and $F$ be closed subsets of $\Delta(A)$ and $\Delta(B)$, respectively. Then

$$
j(E) \otimes B+A \otimes j(F) \subseteq \overline{j(E \times F)} .
$$

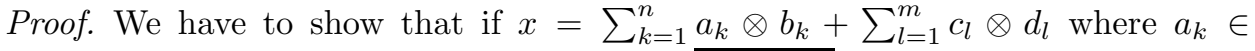
$j(E), b_{k} \in B, c_{l} \in A$ and $d_{l} \in j(F)$, then $x \in \overline{j(E \times F)}$. Of course, we can assume that $a_{k} \neq 0$ for all $k$ and $d_{l} \neq 0$ for all $l$. Since $A$ and $B$ are Tauberian, given $\epsilon>0$, there exist $v_{k} \in B$ and $u_{l} \in A$ such that $\widehat{v_{k}}$ and $\widehat{u_{l}}$ have compact support in $\Delta(B)$ and $\Delta(A)$, respectively, and

$$
\left\|v_{k}-b_{k}\right\| \leq \frac{\epsilon}{2 n\left\|a_{k}\right\|} \text { and }\left\|u_{l}-c_{l}\right\| \leq \frac{\epsilon}{2 m\left\|d_{l}\right\|}
$$

$(1 \leq k \leq n, 1 \leq l \leq m)$. Let

$$
y=\sum_{k=1}^{n} a_{k} \otimes v_{k}+\sum_{l=1}^{m} u_{l} \otimes d_{l} .
$$

Then $\|y-x\| \leq \epsilon$ and $\widehat{y}$ has compact support and vanishes on a neighbourhood of $E \times F$. Thus $y \in j(E \times F)$, and since $\epsilon>0$ was arbitrary, we conclude that $x \in \overline{j(E \times F)}$.

Theorem 1.6. Let $A$ and $B$ be regular commutative Banach algebras such that $A \widehat{\otimes} B$ is semisimple and $A$ and $B$ are Tauberian. Let $E$ and $F$ be closed subsets of $\Delta(A)$ and $\Delta(B)$, respectively. If $E$ and $F$ are weak spectral sets, then $E \times F$ is a weak spectral set for $A \widehat{\otimes} B$. In particular, if $E$ and $F$ are spectral sets, then $E \times F$ is a spectral set.

Proof. Let $m, n \in \mathbb{N}$ be such that $k(E)^{m} \subseteq \overline{j(E)}$ and $k(F)^{n} \subseteq \overline{j(F)}$. Let $I$ be the closed ideal

$$
I=\overline{j(E) \otimes B+A \otimes j(F)},
$$

and note that $h(I)=E \times F$. Since $A$ and $B$ are Tauberian and $A \widehat{\otimes} B$ is semisimple, Lemma 1.5 implies that $I=\overline{j(E \times F)}$. Therefore, to establish the theorem, it suffices to show that $k(E \times F)^{m n} \subseteq I$. 
Let $K$ denote the closed ideal generated by $k(F)^{n}$, and write $\widetilde{F}$ instead of $F$ when $F$ is identified with $\Delta(B / k(F))$. Then $A \widehat{\otimes} K \subseteq \overline{A \otimes j(F)} \subseteq I$. Moreover, with $J=A \widehat{\otimes} k(F)$,

$$
(A \widehat{\otimes} B) / J=A \widehat{\otimes}(B / k(F)) \text { and } \Delta((A \widehat{\otimes} B) / J)=\Delta(A) \times \widetilde{F} .
$$

We apply Corollary 1.4 to $A \widehat{\otimes}(B / k(F))$ and $E \times \widetilde{F}=E \times \Delta(B / k(F))$. If $x \in k(E \times F)$, then $x+J \in k(E \times \widetilde{F})$ and hence

$$
x^{m}+J \in k(E \times \widetilde{F})^{m} \subseteq \overline{j(E \times \widetilde{F})} .
$$

Now, $(j(E) \otimes B+J) / J$ is an ideal of $(A \widehat{\otimes} B) / J$ with hull equal to $E \times \widetilde{F}$. Since $(A \widehat{\otimes} B) / J$ is semisimple and regular, we obtain that

$$
k(E \times F)^{m} \subseteq \overline{j(E) \otimes B+A \otimes k(F)} .
$$

For $a \in j(E), b \in B, c \in A$ and $d \in k(F)$, we have

$$
\begin{aligned}
(a \otimes b+c \otimes d)^{n} & =\sum_{l=1}^{n}\left(\begin{array}{c}
n \\
l
\end{array}\right)\left(a^{l} \otimes b^{l}\right)\left(c^{n-l} \otimes d^{n-l}\right) \\
& =c^{n} \otimes d^{n}+\sum_{l=1}^{n}\left(\begin{array}{c}
n \\
l
\end{array}\right)\left(a^{l} c^{n-l} \otimes b^{l} d^{n-l}\right) \\
& \subseteq A \otimes k(F)^{n}+j(E) \otimes B .
\end{aligned}
$$

It follows that

$$
k(E \times F)^{m n} \subseteq \overline{A \otimes K+j(E) \otimes B} \subseteq I,
$$

as required.

Thus, especially, if $A$ and $B$ are unital regular commutative Banach algebras such that $A \widehat{\otimes} B$ is semisimple and $E$ and $F$ are closed subsets of $\Delta(A)$ and $\Delta(B)$, respectively, then $E \times F$ is a (weak) spectral set if and only if $E$ and $F$ are (weak) spectral sets. It is surprising that Theorem 1.6 (and even Theorem 1.2) has been shown before only in very special cases (see [1, Corollary 2.2 and Lemma 2.3] and [8, Theorem 2.1]).

We finish this section with the observation that it is not true in general that if $E$ and $F$ are weak spectral sets, then $\xi(E \times F)=\xi(E) \xi(F)$. In fact, by [12] for the $n$-dimensional sphere in $\mathbb{R}^{n+1}=\Delta\left(L^{1}\left(\mathbb{R}^{n+1}\right)\right)$ one has $\xi\left(S^{n}\right)=\left\lfloor\frac{n}{2}+1\right\rfloor$, whereas for $S^{n} \times S^{m} \subseteq \mathbb{R}^{n+1} \times \mathbb{R}^{m+1}=\Delta\left(L^{1}\left(\mathbb{R}^{n+1}\right) \widehat{\otimes} L^{1}\left(\mathbb{R}^{m+1}\right)\right), \xi\left(S^{n} \times S^{m}\right) \leq\left\lfloor\frac{n+m}{2}+1\right\rfloor$ by [5, p. 154, Theorem 2].

\section{WEAK SPECTRAL SYNTHESIS}

Of course, if weak synthesis or synthesis holds for $A \widehat{\otimes} B$, then the same is true for $A$ and $B$ (Theorem 1.2). The converse fails in general. For instance, as indicated in [8], it follows from Varopoulos' work [13] that if $G$ is any infinite compact abelian group, then weak synthesis fails for $C(G) \widehat{\otimes} C(G)$. However, if $X$ is a compact Hausdorff space and $B$ is a unital semisimple regular commutative Banach algebra such that $\Delta(B)$ is scattered and, in addition, $B$ satisfies some strong Ditkin type condition, then $C(X) \widehat{\otimes} B$ has synthesis [1, Theorem 2.4]. We establish a weak spectral synthesis result of a similar nature under the hypothesis that $\Delta(B)$ is discrete, but $A$ is arbitrary (Theorem 2.2). 
Lemma 2.1. Let $A$ and $B$ be regular commutative Banach algebras, and suppose that $A \widehat{\otimes} B$ is semisimple and that $\Delta(B)$ is discrete and infinite. If weak synthesis holds for $A \widehat{\otimes} B$, then

$$
\sup \{\xi(E): E \subseteq \Delta(A) \text { closed }\}<\infty .
$$

Proof. Towards a contradiction, assume that there are closed subsets $E_{i}$ of $\Delta(A)$, $i \in \mathbb{N}$, such that the sequence $\left(\xi\left(E_{i}\right)\right)_{i}$ is unbounded. Since $\Delta(B)$ is infinite, we can choose pairwise different elements $\psi_{i}$ of $\Delta(B), i \in \mathbb{N}$. Let $S=\bigcup_{i=1}^{\infty}\left(E_{i} \times\left\{\psi_{i}\right\}\right)$. Then $S$ is closed in $\Delta(A \widehat{\otimes} B)$ since each of the sets $\Delta(A) \times\{\psi\}, \psi \in \Delta(B)$, is open.

For each $i$, let $r_{i}=\xi\left(E_{i}\right)-1$ and choose $a_{i} \in k\left(E_{i}\right)$ such that $a_{i}^{r_{i}} \notin \overline{j\left(E_{i}\right)}$ and $b_{i} \in B$ such that $\psi_{i}\left(b_{i}\right)=1$ and $\psi\left(b_{i}\right)=0$ for all $\psi \neq \psi_{i}$ (such $b_{i}$ exist since $B$ is regular and $\Delta(B)$ is discrete). Replacing $a_{i}$ with a suitable multiple of itself, we can arrange for $\sum_{i=1}^{\infty}\left\|a_{i}\right\| \cdot\left\|b_{i}\right\|<\infty$. Now, let

$$
x=\sum_{i=1}^{\infty} a_{i} \otimes b_{i} \in A \widehat{\otimes} B .
$$

Then $x \in k(S)$ since, for every $\varphi \in E_{i}$,

$$
\varphi \widehat{\otimes} \psi_{i}(x)=\sum_{l=1}^{\infty} \varphi\left(a_{l}\right) \psi_{i}\left(b_{l}\right)=\varphi\left(a_{i}\right)=0 .
$$

Since $S$ is a weak spectral set, $x^{m} \in \overline{j(S)}$ for some $m \in \mathbb{N}$. Since $B$ is semisimple and $\psi\left(b_{i}\right)=0$ for $\psi \neq \psi_{i}$ and $\psi_{i}\left(b_{i}\right)=1(i \in \mathbb{N})$, the elements $b_{i}$ are pairwise orthogonal idempotents. This implies that

$$
\sum_{i=1}^{\infty} a_{i}^{m} \otimes b_{i}=\left(\sum_{i=1}^{\infty} a_{i} \otimes b_{i}\right)^{m}=x^{m} \in \overline{j(S)} .
$$

Fix $i \in \mathbb{N}$. Since $\overline{j(S)} \subseteq \overline{j\left(E_{i} \times\left\{\psi_{i}\right\}\right)}$ and, for $l \neq i$,

$$
a_{l}^{m} \otimes b_{l} \in k\left(\Delta(A) \times\left\{\psi_{i}\right\}\right) \subseteq j\left(E_{i} \times\left\{\psi_{i}\right\}\right),
$$

we conclude that $a_{i}^{m} \otimes b_{i} \in \overline{j\left(E_{i} \times\left\{\psi_{i}\right\}\right)}$. This in turn implies that $a_{i}^{m} \in \overline{j\left(E_{i}\right)}$ (Lemma 1.1), whence $m \geq r_{i}+1=\xi\left(E_{i}\right)$. Since this holds for all $i$, we have reached a contradiction.

Theorem 2.2. Let $A$ and $B$ be regular commutative Banach algebras, and suppose that $A \widehat{\otimes} B$ is semisimple and satisfies Ditkin's condition at infinity. In addition, let $\Delta(B)$ be discrete and $B$ be infinite dimensional. Then the following conditions are equivalent:

(i) weak spectral synthesis holds for $A \widehat{\otimes} B$;

(ii) $\sup \{\xi(E): E \subseteq \Delta(A)$ closed $\}<\infty$.

Moreover, in this case, $\xi(T) \leq \sup \{\xi(E): E \subseteq \Delta(A)$ closed $\}<\infty$ for every closed subset $T$ of $\Delta(A \widehat{\otimes} B)$. In particular, spectral synthesis holds for $A \widehat{\otimes} B$ if and only if spectral synthesis holds for $A$.

Proof. Notice that since $B$ is semisimple, $\Delta(B)$ is finite if and only if $B$ is finite dimensional. Thus (i) $\Rightarrow$ (ii) follows from Lemma 2.1. Thus, suppose that (ii) holds, and let $r$ be the supremum in (ii). Let $T$ be a closed subset of $\Delta(A \widehat{\otimes} B)$ and write

$$
T=\bigcup_{\psi \in \Delta(B)}\left(E_{\psi} \times\{\psi\}\right)
$$


where $E_{\psi} \subseteq \Delta(A)$. Then every $E_{\psi}$ is a closed subset of $\Delta(A)$, and hence $a^{r} \in \overline{j\left(E_{\psi}\right)}$ for all $a \in k\left(E_{\psi}\right)$.

We are going to show that $x^{r} \in \overline{j(T)}$ for each $x \in k(T)$. To that end, recall first that since $A \widehat{\otimes} B$ satisfies Ditkin's condition at infinity, given any $\epsilon>0$, there exists $y \in A \widehat{\otimes} B$ such that $\|x-x y\| \leq \epsilon$ and $\widehat{y}$ has compact support. Therefore it suffices to show that $x^{r} \in \overline{j(T)}$ for $x \in k(T)$ such that $\widehat{x}$ has compact support. Let, say,

$$
\operatorname{supp} \widehat{x}=\bigcup_{j=1}^{n}\left(S_{j} \times\left\{\psi_{j}\right\}\right),
$$

where $\psi_{j} \in \Delta(B)$ and $S_{j} \subseteq \Delta(A)$ is compact, $1 \leq j \leq n$. Now, since $A \widehat{\otimes} B$ is regular and semisimple, there exists a partition of unity associated to these data; that is, there are $u_{1}, \ldots, u_{n} \in A \widehat{\otimes} B$ such that $\operatorname{supp} \widehat{u_{j}} \subseteq \Delta(A) \times\left\{\psi_{j}\right\}$ and $x=\sum_{j=1}^{n} x u_{j}$. It suffices to show that $\left(x u_{j}\right)^{r} \in \overline{j(T)}$ for $1 \leq j \leq n$. Thus we can henceforth assume that

$$
x \in k\left(\left(E_{\psi} \times\{\psi\}\right) \cup(\Delta(A) \times(\Delta(B) \backslash\{\psi\}))\right) .
$$

Define closed ideals $J$ of $B$ and $I$ of $A \widehat{\otimes} B$ by $J=k(\Delta(B) \backslash\{\psi\})$ and

$$
I=k(\Delta(A \widehat{\otimes} B) \backslash(\Delta(A) \times\{\psi\}))=k(\Delta(A) \times(\Delta(B) \backslash\{\psi\})) .
$$

Then $\Delta(I)=\Delta(A) \times\{\psi\}$ and $I=A \widehat{\otimes} k(\Delta(B) \backslash\{\psi\})$ (Lemma 1.3). So the element $x \in I$ can be written as $x=\sum_{i=1}^{\infty} a_{i} \otimes b_{i}$ where $a_{i} \in A$ and $b_{i} \in k(\Delta(B) \backslash\{\psi\})$. Since $B$ is semisimple, $J$ is one-dimensional, and therefore $b_{i}=\alpha_{i} b$ for some $b \in B$ with $\psi(b)=1$ and $\alpha_{i} \in \mathbb{C}(i \in \mathbb{N})$. Hence $x=a \otimes b$ where $a=\sum_{i=1}^{\infty} \alpha_{i} a_{i}$. Now, $a \in k\left(E_{\psi}\right)$ since $x \in k\left(E_{\psi} \times\{\psi\}\right)$ and $\psi(b) \neq 0$. It follows that $a^{r} \in \overline{j\left(E_{\psi}\right)}$.

Finally, $x^{r}=(a \otimes b)^{r}=a^{r} \otimes b$ since $b$ is an idempotent. Since $\widehat{x}$ vanishes on $\Delta(A) \times(\Delta(B) \backslash\{\psi\})$ and $a^{r} \in \overline{j\left(E_{\psi}\right)}$, it follows that $x^{r} \in \overline{j(T)}$.

In Theorem 2.2 we have assumed $B$ to be infinite dimensional. It is obvious from the proofs of Lemma 2.1 and Theorem 2.2 that if $B$ is finite dimensional, then (weak) synthesis holds for $A \widehat{\otimes} B$ if and only if it holds for $A$.

Let $G$ be a locally compact group and $A$ a semisimple regular commutative Banach algebra. Then $L^{1}(G, A)$, the Banach convolution algebra of all $A$-valued integrable functions on $G$, is a semisimple regular commutative Banach algebra that is isomorphic to the projective tensor product $L^{1}(G) \widehat{\otimes} A$. Theorem 2.2 now allows a complete solution to the problem of when (weak) spectral synthesis holds for $L^{1}(G, A)$.

Corollary 2.3. Let $G$ be an infinite locally compact abelian group and $A$ a semisimple regular commutative Banach algebra that is Tauberian and has a bounded approximate identity. Then weak spectral synthesis holds for $L^{1}(G, A)$ if and only if $G$ is compact and

$$
\sup \{\xi(E): E \subseteq \Delta(A) \text { closed }\}<\infty .
$$

In particular, spectral synthesis holds for $L^{1}(G, A)$ if and only if spectral synthesis holds for A.

Proof. If weak spectral synthesis holds for $L^{1}(G, A)$, then it holds for $L^{1}(G)$. However, this forces $G$ to be compact [8, Theorem 3.2]. So $\Delta\left(L^{1}(G)\right)$, which is homeomorphic with the dual group of $G$, is discrete. Hence, by Lemma 2.1, $\sup \{\xi(E): E \subseteq \Delta(A)$ closed $\}<\infty$. 
For the converse, according to Theorem 2.2, it suffices to observe that if $A$ is as in the corollary, then $L^{1}(G, A)$ satisfies Ditkin's condition at infinity. Now, $B=L^{1}(G)$ is Tauberian and has a bounded approximate identity. It is well known that then $A \widehat{\otimes} B$ has a bounded approximate identity. Also, it is obvious that $A \widehat{\otimes} B$ is Tauberian (compare the proof of Lemma 1.5). These two properties together imply that $A \widehat{\otimes} B$ satisfies Ditkin's condition at infinity.

Example 2.4. (i) Concerning the boundedness condition for the set of values $\xi(E)$, it is worth pointing out that for each $n \in \mathbb{N}_{0}$, one can find a natural Banach function algebra $A$ such that

$$
\sup \{\xi(E): E \subseteq \Delta(A) \text { closed }\}=n+1 .
$$

To see this, let $A=C^{(n)}[0,1]$, the algebra of all $n$-times continuously differentiable functions on the interval $[0,1]$. For $t \in[0,1]$ and $0 \leq m \leq n$, let

$$
P_{t, m}=\left\{f \in A: f^{(j)}(t)=0 \text { for } 0 \leq j \leq m\right\} .
$$

Then the map $t \rightarrow \varphi_{t}$, where $\varphi_{t}(f)=f(t)$, is a homeomorphism between $[0,1]$ and $\Delta(A)$ and, due to work of Shilov [10] (see [9, Appendix A 2.4)],

(1) the ideals $P_{t, m}$ are exactly the closed primary ideals of $A$ (that is, the closed ideals $J$ of $A$ with $h(J)$ a singleton),

(2) every closed ideal $I$ of $A$ is the intersection of the primary ideals containing I.

Now, let $E$ be any closed subset of $[0,1]=\Delta(A)$. Then $k(E)^{n} \subseteq \overline{j(E)}$. Indeed, it follows from (1) and (2) that

$$
\overline{j(E)}=\bigcap\left\{P_{t, m}: t \in E, 0 \leq m \leq n\right\} .
$$

Moreover, if $f(t)=0$, then $\left(f^{n+1}\right)^{(j)}(t)=0$ for $0 \leq j \leq n$ as is easily seen by induction on $n$ using the Leibniz rule.

On the other hand, taking for $E$ a singleton, it is clear that $n+1$ is the smallest possible integer $k$ such that $f^{k} \in \overline{j(E)}$ for each $f \in k(E)$.

(ii) Let $M$ be the so-called Mirkil algebra, a certain convolution algebra, which has been used to produce several counterexamples in spectral synthesis. Although $\Delta(M)$ is discrete, spectral synthesis fails in $M$, but as shown in [15, Theorem 1.4], $\xi(E) \leq 2$ for each subset $E$ of $\Delta(M)$.

\section{REFERENCES}

[1] A. Atzmon, Spectral synthesis in regular Banach algebras, Israel J. Math. 8 (1970), 197-212. MR 41:8932

[2] W. Badé and P.C. Curtis, Jr., The Wedderburn decomposition of commutative Banach algebras, Amer. J. Math. 82 (1960), 851-866. MR 23:A529

[3] P. Enflo, A counterexample to the approximation problem in Banach spaces, Acta Math. 130 (1973), 309-317. MR 53:6288

[4] B.R. Gelbaum, Tensor products of Banach algebras, Canad. J. Math. 11 (1959), 297-310. MR 21:2922

[5] W. Kirsch and D. Müller, On the synthesis problem for orbits of Lie groups in $\mathbb{R}^{n}$, Ark. Mat. 18 (1980), 145-155. MR 82f:43006

[6] R. Larsen, Banach algebras, Marcel Dekker, New York, 1973. MR 58:7010

[7] H. Milne, Banach space properties of uniform algebras, Bull. London Math. Soc. 4 (1972), 323-326. MR 48:847

[8] K. Parthasarathy and S. Varma, On weak spectral synthesis, Bull. Austral. Math. Soc. 43 (1991), 279-282. MR 92d:43007

[9] C.E. Rickart, General theory of Banach algebras, D. van Nostrand, 1960. MR 22:5903 
[10] G.E. Shilov, On regular normed rings, Trav. Inst. Math. Steklov (Moscow) 21 (1947), 118 pp. (Russian. English summary). MR 9:596a

[11] J. Tomiyama, Tensor products of commutative Banach algebras, Tohoku Math. J. (2) 12 (1960), 147-154. MR 22:5910

[12] N.Th. Varopoulos, Spectral synthesis on spheres, Proc. Cambridge Philos. Soc. 62 (1966), 379-387. MR 34:1786

[13] N.Th. Varopoulos, Tensor algebras and harmonic analysis, Acta Math. 119 (1967), 51-111. MR 39:1911

[14] C.R. Warner, Weak spectral synthesis, Proc. Amer. Math. Soc. 99 (1987), 244-248. MR $88 \mathrm{c}: 43005$

[15] C.R. Warner, Spectral synthesis in the Mirkil algebra, J. Math. Anal. Appl. 167 (1992), 176-181. MR 93i:46092

Institut für Mathematik, Universität Paderborn, D-33095 Paderborn, Germany

E-mail address: kaniuth@math.uni-paderborn.de 